\title{
Metagenomic Next-Generation Sequencing Improves the Prognosis of Patients with Infectious Diseases on Mechanical Ventilation in the Intensive Care Unit (ICU)
}

\section{Yin Xi}

Guangzhou Institute of Respiratory Disease Jing Zhou

Guangzhou Institute of Respiratory Disease

Zhimin Lin

Guangzhou Institute of Respiratory Disease

Weibo Liang

Guangzhou Institute of Respiratory Disease

Chun Yang

Guangzhou Institute of Respiratory Disease

Dongdong Liu

Guangzhou Institute of Respiratory Disease

Yonghao Xu

Guangzhou Institute of Respiratory Disease Lingbo Nong

Guangzhou Institute of Respiratory Disease

Sibei Chen

Guangzhou Institute of Respiratory Disease

\section{Yuheng Yu}

Guangzhou Institute of Respiratory Disease

Weiqun $\mathrm{He}$

Guangzhou Institute of Respiratory Disease

Jie Zhang

Guangzhou Institute of Respiratory Disease

Rong Zhang

Guangzhou Institute of Respiratory Disease

Ying Pan

Guangzhou Institute of Respiratory Disease

Jieyi Pan

Guangzhou Institute of Respiratory Disease

Jianchun Li

Guangzhou Institute of Respiratory Disease

Qiang Chen 
Guangzhou Institute of Respiratory Disease

Xiaoqing Liu

Guangzhou Institute of Respiratory Disease

Ling Sang ( $\square$ sonysang999@aliyun.com )

Guangzhou Institute of Respiratory Disease

Yuanda Xu

Guangzhou Institute of Respiratory Disease

Yimin Li

Guangzhou Institute of Respiratory Disease

\section{Research}

Keywords: mNGS, ICU, Severe pneumonia, prognosis, pathogens diagnosis, mortality

Posted Date: December 11th, 2020

DOI: https://doi.org/10.21203/rs.3.rs-125410/v1

License: (c) This work is licensed under a Creative Commons Attribution 4.0 International License. Read Full License 


\section{Abstract}

Background: Metagenomic Next-Generation Sequencing (mNGS) has gradually shown its advantages in pathogen identification for clinical infectious disease. However, few studies were conducted on the evaluation between this technique and conventional methods like culture and PCR and the prognosis of patients with infectious diseases on mechanical ventilation in ICU

Methods: We conducted this retrospective study from March 2018 to May 2020 in the first Affiliated Hospital of Guangzhou Medical University, a total of 228 patients with suspected infectious diseases on mechanical ventilation were included, including 104 cases of mNGS group and 124 cases of non-mNGS. Statistical analyses were performed between the two groups and subgroup of whether were immunocompromised. The concordance between mNGS, culture and PCR was also assessed.

Results: The 28-day mortality rate of the patients in the mNGS group was lower after the baseline difference correction (19.23\% vs. $29.03 \% \unrhd \mathrm{p}=0.039)$, indicating that mNGS may improve the prognosis of patients in ICU. And subgroup analysis showed that mNGS could improve the 28-day mortality of nonimmunosuppressive patients $\varangle 14.06 \%$ vs. $29.82 \%, p=0.018$. According to the analysis of Logistic Regression, not performing mNGS, high APACHE II score and hypertension were independent risk factors for 28-day mortality, which strongly suggested that mNGS was one of the key factors affecting prognosis. A total of 157 samples performed mNGS, 116 of them received both mNGS and culture. mNGS presented advantages of positivity $(69.8 \%$ double positive and $25.0 \%$ mNGS positive only) and concordance ( $79.0 \%$, match and partly match).

Conclusions: mNGS may improve the prognosis and reduce the 28-day mortality rate of patients with infectious diseases on mechanical ventilation in ICU. This technique has shown its advantages comparing with conventional methods, and will be wildly used as a promising technology for infectious disease.

\section{Introduction}

Metagenomic next-generation sequencing (mNGS) is increasingly being applied in clinical laboratories for unbiased culture-independent diagnosis. Whether it can be a next routine pathogen identification tool has become a topic of concern. We review the current implementation of this new technology for infectious disease diagnostics and discuss the feasibility of transforming mNGS into a routine diagnostic test. Since 2008, numerous studies from over 20 countries have revealed the practicality of mNGS in the work-up of undiagnosed infectious diseases. mNGS performs well in identifying rare, novel, difficult-to-detect and coinfected pathogens directly from clinical samples and presents great potential in resistance prediction by sequencing the antibiotic resistance genes, providing new diagnostic evidence that can be used to guide treatment options and improve antibiotic stewardship. Many physicians recognized mNGS as a last resort method to address clinical infection problems. Although several hurdles, such as workflow validation, quality control, method standardisation, and data interpretation, remain before mNGS can be implemented routinely in 
clinical laboratories, they are temporary and can be overcome by rapidly evolving technologies. With more validated workflows, lower cost and turnaround time, and simplified interpretation criteria, mNGS will be widely accepted in clinical practice. Overall, mNGS is transforming the landscape of clinical microbiology laboratories, and to ensure that it is properly utilised in clinical diagnosis, both physicians and microbiologists should have a thorough understanding of the power and limitations of this method.

In the intensive care unit (ICU), severe pneumonia diagnosis is particularly complex due to the similar clinical symptoms. It generally leads to the dysfunction of other organs and thus carries high mortality [1-3]. So it is critical to provide an effective symptomatic treatment early. However, the diagnosis is challenging because of the multitude of possible pathogens. Metagenomic next-generation sequencing (mNGS) is a novel approach that can theoretically detect all nucleic acids in a clinical sample. After the first successful clinical application reported in 2014[4], the clinical attention and acceptance of mNGS has been increasing, due to its high sensitivity and wide coverage, it can improve the positive rate compared with the traditional methods, which is very important for the detection of rare pathogens $[5,6]$. In the near future, mNGS might have the potential to become a routine diagnostic workup, especially in the diagnosis of severe pneumonia [7]. At present, literature relevant to clinical applications has mostly emerged as case reports or small-scale cohort studies, most of which have concerned about comparative analysis of infected patients [8-11]. However, reports on the relationship between infection and immunosuppression in patients with severe pneumonia remain scarce. In this study, we will discuss the performance of mNGS in clinical practice. It will include the composition of pathogens in the patients of severe pneumonia under the premise of immunosuppression or not, so as to provide clinical basis for early empirical antibiotic treatment, also include the comparison of clinical detection methods of Pneumocystis, and the correlation between mNGS and prognosis of patients.

\section{Methods}

\section{Patients of This Study}

This retrospective study was conducted between March 2018 and May 2020. We reviewed the medical records of patients admitted to the intensive care unit (ICU) of the First Affiliated Hospital of Guangzhou Medical University. Patients with infectious diseases on mechanical ventilation were admitted to ICU during this period were included or excluded for our study according to the following criteria.

Inclusion:

1)more than 18 years of age;

2)patients with mechanical ventilation, the expected mechanical ventilation duration was more than 48 hours [2];

3)patients suspected of infectious disease requiring microbiological investigations $[2,11]$.

Exclusion:

1)pregnant woman; 
2)patient unable to fulfill the required medical follow-up;

3)patients participating in a clinical trial implicating a new drug;

A total of 246 patients were enrolled, including 112 in the mNGS group and 134 in the non-mNGS group. While 8 cases in mNGS group and 10 cases in non-mNGS group were excluded due to incomplete data. 50 of the remaining 228 cases were considered as immunocompromised when clinically diagnosed as:

1)individuals on immunosuppressive therapy (e.g., cytotoxic agents, glucocorticoids, etc.);

2)transplantation individuals (e.g., solid organ transplantation or bone marrow transplantation);

3)hematologic cancers;

4)certain trauma or surgery (e.g., splenectomy);

5) secondary to metabolic diseases (e.g., malnutrition, non-controlled diabetes, uremia).

All 228 cases have complete medical treatment record including Clinical diagnosis and symptoms, APACHE II score, SOFA score, comorbidities and Blood laboratory tests when transferred to ICU, 28-day mortality, Inhospital mortality, Length of ICU stay, Days ventilator-free at day 28, and Total cost. For details see supplementary excel 1. The Major diagnosis of ICU admission is Severe pneumonia $(n=198), \operatorname{ARDS}(n=107)$, Septic shock $(n=36)$, and Acute kidney injury $(n=27)$.

Statistically analysis was performed based on whether performed mNGS or whether immunocompromised. Since the data were anonymous, there is no need for informed consent. The flowchart of this comparative study described in Fig. 1.

\section{Metagenomic Next-generation Sequencing and Analysis Nucleic Acid Extraction}

In our study, 104 cases used blood (41), bronchoalveolar lavage fluid (BALF,80), sputum (31), cerebrospinal fluid (1); and pleural effusion (4) to perform mNGS for pathogen identification. Blood samples were drawn from patients into ethylenediaminetetraacetic acid tubes with a volume of $5 \mathrm{ml}$, then stored at $4{ }^{\circ} \mathrm{C}$. The plasma separation experiment should be completed within 8 hours, with the method of centrifugation at $1600 \mathrm{~g}$ for $10 \mathrm{~min}$, the supernatant was transferred to new sterile tubes for DNA extraction. Samples of 1-4 ml of sputum, bronchoalveolar lavage fluid, pleural effusion were collected from patients according to standard protocols and stored in $-80{ }^{\circ} \mathrm{C}$ before DNA extraction. For sputum samples, $0.1 \%$ dithiothreitol (DTT) was used for liquefying at room temperature for $30 \mathrm{~min}$. BALF and pleural effusion could go directly to the DNA extraction procedures.

To begin with DNA extraction, $0.5 \mathrm{ml}$ of the samples was transferred to a new $1.5 \mathrm{ml}$ microcentrifuge tube, DNA was extracted using the MAPMI Sample preparation kit (360120, CapitalBio Corporation, Beijing, China) according to the manufacturer's recommendation.

\section{Library Preparation}


The extracted DNA was quantified before the preparation of sequencing libraries with a total DNA less than $500 \mathrm{ng}$. DNA libraries were constructed through end repair, adapters adding, and PCR amplification. The quality of the DNA libraries was assessed using an Agilent 2100 Bioanalyzer (Agilent Technologies, Santa Clara, California) combined with qPCR based on Applied Biosystems 7500 Real-Time PCR System (Thermo Fisher, USA), the primers based on the sequences of the adapters were used for the qPCR. Then quantified DNA libraries were pooled and analyzed on BioelectronSeq 4000 (CapitalBio Corporation, Beijing, China) sequencing platform. The whole process requires $48 \sim 72$ hours from DNA extraction to issuing the reports.

\section{Bioinformatic Analysis}

The original sequencing data were subjected for quality control, and the reads with length less than $50 \mathrm{bp}$, lowquality, and low complexity were removed. The remaining high-quality sequencing data mapped to the human reference genome grch38 for human host sequencing depletion use Bowtie2. Subsequently, the non-human sequences were classified by simultaneous alignment to the databases of viruses, bacteria, fungi and parasites for annotation. The final pathogen detection results include a list of suspected pathogens, the number of hit reads and genome level coverage statistics. All the pathogenic genomic sequences were downloaded from $\mathrm{NCBI}$ and PATRIC databases. At present, the bacterial database contains 13992 species, the fungal database contains 1659 species, the virus database contains 13000 species, and the parasite database contains 287 pathogen genomic data.

In order to judge the suspected pathogens, we reviewed data of different sample type of healthy people, and calculated the relevant reference values, including hit reads number and coverage of all bacteria, fungi, viruses and parasites detected $[6,12,13]$. The quantiles, median and maximum values at $5 \%, 75 \%, 95 \%$ of these samples were calculated, and the $5 \%$ and $95 \%$ quantiles were used as reference ranges for normal people. The criteria for judging suspected pathogens are as follows:

1) the number of hit reads is greater than $95 \%$ quantile in the reference range;

2) the length of target genome covered is greater than $95 \%$ quantile in the reference range;

3) the suspected pathogen has potential infection ability.

\section{Statistical Analyses}

Statistical analyses were performed using R software v.4.0.2. Baseline characteristics were presented as the median (interquartile range) and the count (proportion), then analyzed using the Mann-Whitney $U$ test and the Chi-square test. $P$ values $<0.05$ were considered statistically significant. Since the mNGS group and non-mNGS group had a significant difference in APACHE II score, ARDs, Lymphocyte and LDH $(P<0.1$; Table 1), comparisons of the outcome data (28-day mortality and in-hospital mortality) was corrected using characteristics mentioned above as covariate. A multivariate logistic regression analysis was used for multiple characteristics evaluation on survival.

\section{Result}

1.Comparison of whether to perform $m N G S$ 
In the total 228 patients, 104 of them performed mNGS and 124 patients not. Characteristics and baselines of patients who whether to performed mNGS, statistical analysis result list in Table 1. Statistical analysis information includes age, sex, APACHE II scored and SOFA score, Major diagnosis of ICU admission, Comorbidities, Blood laboratory tests when transferred to ICU. Compared with non-mNGS group, mNGS patients had higher proportion of ARDS (56.7\% vs 38.7\%, $P=0.007)$, lower lymphocytes $(0.4$ vs $0.6, P<0.001)$, higher LDH level (446.3 vs 307.8, P < 0.001) and higher APACHE II score (although there was no statistical difference between the two groups). 
Table 1

Characteristics and baselines of patients who whether to perform mNGS

\begin{tabular}{|c|c|c|c|}
\hline & mNGS(n = 104) & Non-mNGS( $n=124)$ & $p$ value \\
\hline Age (y) & $61.5(47.5 \sim 70)$ & $63(52.8 \sim 71.3)$ & 0.373 \\
\hline \multicolumn{4}{|l|}{ Sex } \\
\hline Female, n(\%) & $30(28.9 \%)$ & $35(28.2 \%)$ & 0.918 \\
\hline Male, $\mathrm{n}(\%)$ & $74(71.2 \%)$ & $89(71.8 \%)$ & \\
\hline \multirow[t]{2}{*}{ APACHE II score } & $21(16.3 \sim 26.8)$ & $19(14 \sim 25)$ & 0.093 \\
\hline & & $\mathrm{n}=121$ & \\
\hline \multirow[t]{2}{*}{ SOFA score } & $9(6 \sim 12)$ & $8(5 \sim 13)$ & 0.653 \\
\hline & & $n=123$ & \\
\hline \multicolumn{4}{|l|}{ Major diagnosis of ICU admission } \\
\hline Severe pneumonia, $\mathrm{n}(\%)$ & $88(84.6 \%)$ & $110(88.7 \%)$ & 0.362 \\
\hline ARDS, n(\%) & $59(56.7 \%)$ & $48(38.7 \%)$ & $0.007 *$ \\
\hline Septic shock, n(\%) & $14(13.5 \%)$ & $22(17.7 \%)$ & 0.377 \\
\hline Acute kidney injury, n(\%) & $8(7.7 \%)$ & $19(15.3 \%)$ & 0.076 \\
\hline \multicolumn{4}{|l|}{ Comorbidities } \\
\hline Hypertension, n(\%) & $29(27.9 \%)$ & $38(30.7 \%)$ & 0.649 \\
\hline Diabetes, n(\%) & $10(9.6 \%)$ & $14(11.3 \%)$ & 0.681 \\
\hline Coronary artery disease, n(\%) & $12(11.5 \%)$ & $16(12.9 \%)$ & 0.754 \\
\hline Rheumatic disease, n(\%) & $9(8.7 \%)$ & $15(12.1 \%)$ & 0.399 \\
\hline Chronic obstructive pulmonary disease, $\mathrm{n}(\%)$ & $18(17.3 \%)$ & $26(21.0 \%)$ & 0.486 \\
\hline Chronic kidney disease, n(\%) & $7(6.7 \%)$ & $6(4.8 \%)$ & 0.539 \\
\hline Chronic liver disease, n(\%) & $2(1.9 \%)$ & $5(4.0 \%)$ & 0.359 \\
\hline Previous history of malignancy, n(\%) & $20(19.2 \%)$ & $29(23.4 \%)$ & 0.447 \\
\hline \multicolumn{4}{|l|}{ Blood laboratory tests when transferred to ICU } \\
\hline \multirow[t]{2}{*}{ White Blood Cell $\left(\times 10^{9} / \mathrm{L}\right)$} & $10.60(6.6 \sim 14.5)$ & $11.30(7.2 \sim 15.2)$ & 0.357 \\
\hline & & $n=123$ & \\
\hline \multirow[t]{2}{*}{ Lymphocyte(× 1099/L) } & $0.4(0.2 \sim 0.6)$ & $0.6(0.4 \sim 1.1)$ & $P<0.001 *$ \\
\hline & & $n=123$ & \\
\hline C-reactive protein(g/L) & & & \\
\hline
\end{tabular}




\begin{tabular}{|llll|}
\hline & mNGS $(\mathrm{n}=104)$ & Non-mNGS$(\mathrm{n}=124)$ & $\boldsymbol{p}$ value \\
\hline Procalcitonin $(\mathrm{ng} / \mathrm{mL})$ & $1.18(0.4 \sim 3.5)$ & $1.12(0.4 \sim 7.2)$ & 0.34 \\
& $\mathrm{n}=96$ & $\mathrm{n}=113$ & \\
\hline $\mathrm{LDH}(\mathrm{U} / \mathrm{L})$ & $446(300.8 \sim 630.0)$ & $308(231.8 \sim 451.7)$ & $\mathrm{P}<0.001^{*}$ \\
& & $\mathrm{n}=122$ & \\
\hline
\end{tabular}

\section{Note}

All data were presented as the median (interquartile range) unless otherwise stated.

$* P<0.05$ was considered statistically significant.

To evaluate the outcomes of patients who whether to perform mNGS, the result of 28-day mortality, In-hospital mortality, Length of ICU stay, Days ventilator-free at day 28 , Total cost, list in Table 2. No significant statistical difference was found in 28-day mortality and in-hospital mortality.

Table 2

Outcomes of patients who whether to perform mNGS

\begin{tabular}{|c|c|c|c|}
\hline & mNGS(n = 104) & Non-mNGS( $n=124)$ & $p$ value \\
\hline 28-day mortality, n(\%) & $20(19.2 \%)$ & $36(29.0 \%)$ & 0.087 \\
\hline In-hospital mortality, n(\%) & $31(29.8 \%)$ & $46(37.1 \%)$ & 0.246 \\
\hline Length of ICU stay & $26.5(14 \sim 57)$ & $14(6.8 \sim 31.3)$ & $P<0.001 *$ \\
\hline \multirow[t]{2}{*}{ Days ventilator-free at day 28} & $5(0 \sim 16)$ & $18.50(2.8 \sim 24)$ & $\mathrm{P}<0.001 *$ \\
\hline & & $\mathrm{n}=98$ & \\
\hline Total cost & 359914(229995 784650) & 197664(102715 404223) & $P<0.001 *$ \\
\hline \multicolumn{4}{|l|}{ Note } \\
\hline \multicolumn{4}{|c|}{${ }^{*} \mathrm{P}<0.05$ was considered statistically significant. } \\
\hline \multicolumn{4}{|c|}{$\begin{array}{l}\text { The results of Table 2, basic clincal information of mNGS patients was not considered. According to } \\
\text { APACHE II score, ARDS proportion, lymphocyte count and LDH level, logistic regression was used for } \\
\text { analysis. The results (Table 3) showed that there were significant differences in 28-day mortality }(19.23 \% \\
\text { vs } 29.03 \%, P=0.039) \text {. }\end{array}$} \\
\hline
\end{tabular}

Table 3

Outcomes of patients who whether to perform mNGS after correction according to baselines

\begin{tabular}{|llll|}
\hline & mNGS$(\mathrm{n}=104)$ & non-mNGS( $\mathrm{n}=124)$ & $p$ value \\
\hline 28-day mortality, $\mathrm{n}(\%)$ & $20(19.2 \%)$ & $36(29.0 \%)$ & $0.039 *$ \\
\hline In-hospital mortality, $\mathrm{n}(\%)$ & $31(29.8 \%)$ & $46(37.1 \%)$ & 0.150 \\
\hline
\end{tabular}




\section{Note}

*P $<0.05$ was considered statistically significant.

The total 228 patients who were survival or non-survival at day 28 , statistical analysis result list in Table 3 . The APACHE II score, SOFA score, AKI proportion, concentration of procalcitonin ( $\mathrm{ng} / \mathrm{ml}$ ) show significant differences between the patient who were survival or non-survival at day 28 , but whether perform mNGS show no significant difference, this is probably because the mNGS test does not be widely used in clinical hospital or this test has not accepted wide recognition because its high cost or other limitations. 
Table 4

Characteristics and baselines of patients who were survival or non-survival at day 28

\begin{tabular}{|c|c|c|c|}
\hline & Survival( $(n=172)$ & Non-survival(n= 56) & $p$ value \\
\hline Age(y) & $63(50.8 \sim 70.3)$ & $62(50 \sim 72.3)$ & 0.688 \\
\hline \multicolumn{4}{|l|}{ Sex } \\
\hline Female, $n(\%)$ & $54(31.4 \%)$ & $11(19.6 \%)$ & 0.091 \\
\hline Male, n(\%) & $118(68.6 \%)$ & $45(80.4 \%)$ & \\
\hline \multirow[t]{2}{*}{ APACHE II score } & $19(15 \sim 24)$ & $25(19 \sim 35)$ & $P<0.001 *$ \\
\hline & $n=169$ & $\mathrm{n}=54$ & \\
\hline \multirow[t]{2}{*}{ SOFA score } & $8(5 \sim 11.8)$ & $12(8 \sim 15.5)$ & $P<0.001 *$ \\
\hline & $n=170$ & & \\
\hline \multicolumn{4}{|l|}{ Major diagnosis of ICU admission } \\
\hline Severe pneumonia, n(\%) & $149(86.6 \%)$ & $49(87.5 \%)$ & 0.867 \\
\hline ARDS, n(\%) & $81(47.1 \%)$ & $26(46.4 \%)$ & 0.931 \\
\hline Septic shock, n(\%) & $26(15.1 \%)$ & $10(17.9 \%)$ & 0.625 \\
\hline Acute kidney injury, n(\%) & $16(9.3 \%)$ & $11(19.6 \%)$ & $0.038 *$ \\
\hline \multicolumn{4}{|l|}{ Comorbidities } \\
\hline Hypertension, n(\%) & $57(33.1 \%)$ & $10(17.9 \%)$ & $0.029 *$ \\
\hline Diabetes, n(\%) & $18(10.5 \%)$ & $6(10.7 \%)$ & 0.958 \\
\hline Coronary artery disease, $\mathrm{n}(\%)$ & $19(11.1 \%)$ & $9(16.1 \%)$ & 0.320 \\
\hline Rheumatic disease, n(\%) & $18(10.5 \%)$ & $6(10.7 \%)$ & 0.958 \\
\hline Chronic obstructive pulmonary disease, $\mathrm{n}(\%)$ & $35(20.4 \%)$ & $9(16.1 \%)$ & 0.481 \\
\hline Chronic kidney disease, n(\%) & $8(4.7 \%)$ & $5(8.9 \%)$ & 0.231 \\
\hline Chronic liver disease, $\mathrm{n}(\%)$ & $4(2.3 \%)$ & $3(5.4 \%)$ & 0.253 \\
\hline Previous history of malignancy, $\mathrm{n}(\%)$ & $40(23.3 \%)$ & $9(16.1 \%)$ & 0.256 \\
\hline \multicolumn{4}{|l|}{ Blood laboratory tests when transferred to ICU } \\
\hline \multirow[t]{2}{*}{ White Blood Cell $\left(\times 10^{9} / \mathrm{L}\right)$} & $11.1(6.9 \sim 15.2)$ & $10.9(5.3 \sim 14.3)$ & 0.364 \\
\hline & $n=171$ & & \\
\hline \multirow[t]{2}{*}{ Lymphocyte $\left(\times 10^{9} / \mathrm{L}\right)$} & $0.5(0.3 \sim 0.9)$ & $0.4(0.28 \sim 0.8)$ & 0.820 \\
\hline & $n=171$ & & \\
\hline
\end{tabular}




\begin{tabular}{|llll|}
\hline & Survival( $\mathbf{n}=172)$ & Non-survival $(\mathrm{n}=56)$ & $\boldsymbol{p}$ value \\
\hline Procalcitonin $(\mathrm{ng} / \mathrm{mL})$ & $0.9(0.3 \sim 3.5)$ & $4.1(0.6 \sim 9.0)$ & 0.004* $^{*}$ \\
& $\mathrm{n}=159$ & $\mathrm{n}=50$ & \\
\hline LDH(U/L) & $340(246.0 \sim 479.8)$ & $509(344.9 \sim 685.0)$ & $\mathrm{P}<0.001^{*}$ \\
\hline Perform mNGS, $\mathrm{n}(\%)$ & $84(48.8 \%)$ & $20(35.7 \%)$ & 0.087 \\
\hline
\end{tabular}

\section{Note}

${ }^{*} \mathrm{P}<0.05$ was considered statistically significant.

From table above, we select the indices present $\mathrm{P}<0.1$ for logistic regression. The result show that without performing mNGS, higher APACHA II score and Hypertension may be high risk factors, with the $\operatorname{Pr}(>|z|) 0.007$, 0.027 and 0.036 separately.

Table 5

Logistic regression of indices $\mathrm{P}<0.1$ in table

\begin{tabular}{|lllll|}
\hline & Estimate & Std. Error & $\mathbf{z}$ value & $\operatorname{Pr}(>|\mathbf{z}|)$ \\
\hline APACHE.II.score & $7.5 \mathrm{E}-02$ & $2.8 \mathrm{E}-02$ & 2.68 & $\mathbf{0 . 0 0 7 *}$ \\
\hline SOFA.score & $4.4 \mathrm{E}-02$ & $5.4 \mathrm{E}-02$ & 0.81 & 0.419 \\
\hline Acute.kidney.injury & $-1.6 \mathrm{E}-02$ & $5.5 \mathrm{E}-01$ & -0.03 & 0.976 \\
\hline Hypertension & $-1.0 \mathrm{E}+00$ & $4.6 \mathrm{E}-01$ & -2.21 & $\mathbf{0 . 0 2 7 *}$ \\
\hline Procalcitonin & $1.8 \mathrm{E}-03$ & $6.4 \mathrm{E}-03$ & 0.29 & 0.773 \\
\hline LDH & $7.5 \mathrm{E}-05$ & $8.1 \mathrm{E}-05$ & 0.93 & 0.352 \\
\hline Perform mNGS & $-8.0 \mathrm{E}-01$ & $3.8 \mathrm{E}-01$ & -2.09 & $\mathbf{0 . 0 3 6 *}$ \\
\hline
\end{tabular}

\section{Note}

*P $<0.05$ was considered statistically significant.

\section{Comparison of whether were Immunocompromised}

Characteristics and baselines of patients who whether were immunocompromised and whether performed mNGS, statistical analysis result shows in Table 6 . The patients immunocompromised counted 50 , and 40 of them performed mNGS. This is possibly because the immunocompromised patients got higher risk of infectious diseases. The evaluation indices in the immunocompromised group, perform mNGS or not shows no significant difference except the LDH level (513.75 vs 344.1, $\mathrm{P}=0.0337)$, as the number of not perform mNGS is only 10 , this result could not explain more.

The nonimmunocompromised patients counted 178 , and 64 of them performed mNGS. The evaluation indices in this group, the proportion of ARDS, Lymphocyte, LDH shows Statistical difference. 
Table 6

Characteristics and baselines of patients who whether were immunocompromised

\begin{tabular}{|c|c|c|c|c|c|c|}
\hline & \multicolumn{3}{|c|}{ Immunocompromised patient } & \multicolumn{3}{|c|}{ Nonimmunocompromised patient } \\
\hline & $\begin{array}{l}\text { mNGS, (n } \\
=40)\end{array}$ & $\begin{array}{l}\text { Non- } \\
\text { mNGS, (n } \\
=10)\end{array}$ & $\begin{array}{l}p \\
\text { value }\end{array}$ & $\begin{array}{l}\operatorname{mNGS}(\mathrm{n}= \\
64)\end{array}$ & $\begin{array}{l}\text { Non-mNGS, } \\
(n=114)\end{array}$ & $\begin{array}{l}p \\
\text { value }\end{array}$ \\
\hline Age(y) & $\begin{array}{l}55(45 \sim \\
63.3)\end{array}$ & $\begin{array}{l}58.5(53 \sim \\
66.8)\end{array}$ & 0.560 & $\begin{array}{l}66(49 \sim \\
74)\end{array}$ & $\begin{array}{l}63.5(52.3 \sim \\
72)\end{array}$ & 0.657 \\
\hline \multicolumn{7}{|l|}{ Sex } \\
\hline Female, $n(\%)$ & $13(32.5 \%)$ & $3(30 \%)$ & 0.879 & $17(26.6 \%)$ & $32(28.1 \%)$ & 0.829 \\
\hline Male, $n(\%)$ & $27(67.5 \%)$ & $7(70 \%)$ & & $47(73.4 \%)$ & $82(71.9 \%)$ & \\
\hline APACHE II score & $\begin{array}{l}24(19 \sim \\
27.5)\end{array}$ & $\begin{array}{l}18(16.5 \sim \\
25)\end{array}$ & 0.223 & $\begin{array}{l}19(15.5 \sim \\
24.5)\end{array}$ & $19(14 \sim 25)$ & 0.694 \\
\hline SOFA score & $\begin{array}{l}10(7.5 \sim \\
13)\end{array}$ & $\begin{array}{l}8(6.5 \sim \\
9.75)\end{array}$ & 0.179 & $8(6 \sim 11)$ & $9(5 \sim 13)$ & 0.612 \\
\hline \multicolumn{7}{|l|}{$\begin{array}{l}\text { Major diagnosis of ICU } \\
\text { admission }\end{array}$} \\
\hline Severe pneumonia, n(\%) & $34(85 \%)$ & $10(100 \%)$ & 0.192 & $54(84.4 \%)$ & $100(87.7 \%)$ & 0.531 \\
\hline ARDS, n (\%) & $26(65 \%)$ & $7(70 \%)$ & 0.765 & $33(51.6 \%)$ & $41(36.0 \%)$ & 0.043 \\
\hline Septic shock, n(\%) & $4(10 \%)$ & $2(20 \%)$ & 0.384 & $10(15.6 \%)$ & $20(17.5 \%)$ & 0.742 \\
\hline Acute kidney injury, n(\%) & $1(2.5 \%)$ & $1(10 \%)$ & 0.279 & $7(10.9 \%)$ & $18(15.8 \%)$ & 0.371 \\
\hline \multicolumn{7}{|l|}{ Comorbidities } \\
\hline Hypertension, n(\%) & $5(12.5 \%)$ & $2(20 \%)$ & 0.541 & $24(37.5 \%)$ & $36(31.6 \%)$ & 0.423 \\
\hline Diabetes, n(\%) & $3(7.5 \%)$ & $3(30 \%)$ & 0.050 & $7(10.9 \%)$ & $11(9.7 \%)$ & 0.784 \\
\hline $\begin{array}{l}\text { Coronary artery disease, } \\
\mathrm{n}(\%)\end{array}$ & $2(5 \%)$ & $0(0 \%)$ & 0.470 & $10(15.6 \%)$ & $16(14.0 \%)$ & 0.773 \\
\hline Rheumatic disease, n(\%) & $6(15 \%)$ & $7(70 \%)$ & $\begin{array}{l}P< \\
0.001^{*}\end{array}$ & $3(4.7 \%)$ & $8(7.0 \%)$ & 0.536 \\
\hline $\begin{array}{l}\text { Chronic obstructive } \\
\text { pulmonary disease, n(\%) }\end{array}$ & $5(12.5 \%)$ & $0(0 \%)$ & 0.239 & $13(20.3 \%)$ & $26(22.8 \%)$ & 0.699 \\
\hline $\begin{array}{l}\text { Chronic kidney disease, } \\
\mathrm{n}(\%)\end{array}$ & $6(15 \%)$ & $1(10 \%)$ & 0.684 & $1(1.6 \%)$ & $5(4.4 \%)$ & 0.316 \\
\hline Chronic liver disease, $\mathrm{n}(\%)$ & $0(0 \%)$ & $0(0 \%)$ & - & $2(3.1 \%)$ & $5(4.4 \%)$ & 0.678 \\
\hline $\begin{array}{l}\text { Previous history of } \\
\text { malignancy, } \mathrm{n}(\%)\end{array}$ & $11(27.5 \%)$ & $1(10 \%)$ & 0.246 & $9(14.1 \%)$ & $28(24.6 \%)$ & 0.098 \\
\hline
\end{tabular}




\begin{tabular}{|c|c|c|c|c|c|c|}
\hline \multirow[b]{2}{*}{ White Blood Cell $\left(\times 10^{9} / \mathrm{L}\right)$} & \multicolumn{3}{|c|}{ Immunocompromised patient } & \multicolumn{3}{|c|}{ Nonimmunocompromised patient } \\
\hline & $\begin{array}{l}8.5(0.1 \sim ~ \\
14.7)\end{array}$ & $\begin{array}{l}7(6.5 \sim \\
9.8)\end{array}$ & 0.737 & $\begin{array}{l}11.33(0.3 \\
\sim 14.4)\end{array}$ & $\begin{array}{l}11.5(7.7 \sim \\
15.5)\end{array}$ & 0.727 \\
\hline Lymphocyte(× 109/L) & $\begin{array}{l}0.2(0.1 \sim \\
0.4)\end{array}$ & $\begin{array}{l}0.4(0.2 \sim \\
1.2)\end{array}$ & 0.080 & $\begin{array}{l}0.5(0.3 \sim \\
0.7)\end{array}$ & $0.6(0.4 \sim 1)$ & $0.033^{*}$ \\
\hline \multicolumn{7}{|l|}{ C-reactive protein(g/L) } \\
\hline Procalcitonin(ng/mL) & $\begin{array}{l}1.2(0.2 \sim \\
5.8)\end{array}$ & $\begin{array}{l}0.8(0.4 \sim \\
1.7)\end{array}$ & 0.868 & $\begin{array}{l}1.1(0.4 \sim \\
2.5)\end{array}$ & $\begin{array}{l}1.3(0.3 \sim \\
7.3)\end{array}$ & 0.192 \\
\hline $\mathrm{LDH}(\mathrm{U} / \mathrm{L})$ & $\begin{array}{l}514(371.4 \\
\sim 665.7)\end{array}$ & $\begin{array}{l}344(286 \sim \\
404)\end{array}$ & $0.034^{*}$ & $\begin{array}{l}387(263.8 \\
\sim 591.6)\end{array}$ & $\begin{array}{l}303(231 \sim \\
454.3)\end{array}$ & $0.013^{*}$ \\
\hline
\end{tabular}

\section{Note}

${ }^{*} \mathrm{P}<0.05$ was considered statistically significant.

The outcomes of patients who whether were immunocompromised and whether perform mNGS, statistical analysis in Table 7. The proportion of 28 -day mortality shows significant difference $(14.06 \%$ vs $29.82 \%, P=$ 0.018 in Nonimmunocompromised patients. But the subgroup of immunocompromised patients shows no significant difference $(27.5 \%$ vs $20 \%, P=0.629)$.

Table 7

Outcomes of patients who whether were immunocompromised

\begin{tabular}{|c|c|c|c|c|c|c|}
\hline & \multicolumn{3}{|c|}{ Immunocompromised patient } & \multicolumn{3}{|c|}{ Nonimmunocompromised patient } \\
\hline & $\mathrm{mNGS}(\mathrm{n}=40)$ & $\begin{array}{l}\text { Non-mNGS }(\mathrm{n}= \\
10)\end{array}$ & $\begin{array}{l}p \\
\text { value }\end{array}$ & mNGS(n = 64) & $\begin{array}{l}\text { Non-mNGS, (n } \\
=114)\end{array}$ & $\begin{array}{l}p \\
\text { value }\end{array}$ \\
\hline $\begin{array}{l}\text { 28-day } \\
\text { mortality, } \\
\mathrm{n}(\%)\end{array}$ & $11(27.5 \%)$ & $2(20 \%)$ & 0.629 & $9(14.1 \%)$ & $34(29.8 \%)$ & $0.018 *$ \\
\hline $\begin{array}{l}\text { In- } \\
\text { hospital } \\
\text { mortality, } \\
\text { n(\%) }\end{array}$ & $14(35 \%)$ & $4(40 \%)$ & 0.769 & $17(26.6 \%)$ & $42(36.8 \%)$ & 0.162 \\
\hline $\begin{array}{l}\text { Length of } \\
\text { ICU stay }\end{array}$ & $24.5(16.8 \sim 41)$ & $17(8 \sim 49.3)$ & 0.235 & $30.5(14 \sim 63)$ & $14(5.25 \sim 29)$ & $\begin{array}{l}P< \\
0.001 *\end{array}$ \\
\hline $\begin{array}{l}\text { Days } \\
\text { ventilator- } \\
\text { free at } \\
\text { day } 28\end{array}$ & $4.5(0 \sim 14.3)$ & $19.5(0.8 \sim 21)$ & 0.063 & $5(0 \sim 18)$ & $17.5(3.5 \sim 24)$ & $\begin{array}{l}P< \\
0.001 *\end{array}$ \\
\hline Total cost & $\begin{array}{l}352448(248186 \\
\sim 622993)\end{array}$ & $\begin{array}{l}363710(307961 \\
\sim 636735)\end{array}$ & 0.877 & $\begin{array}{l}402835(215034 \\
\sim 805246)\end{array}$ & $\begin{array}{l}170727(98553 \\
\sim 361456)\end{array}$ & $\begin{array}{l}\mathrm{P}< \\
0.001 *\end{array}$ \\
\hline
\end{tabular}

Note

$* \mathrm{P}<0.05$ was considered statistically significant. 
Characteristics and baselines of patients who were survival or non-survival at day 28 , immunocompromised or not, perform mNGS or not. statistical analysis base on indices of clinical factor list in Table 8.

In the subgroup immunocompromised of total 50 patients, 37 survived and 13 were non-survival, no statistical difference found based on the indices of clinical factors. Maybe the cases of Immunocompromised patients are not enough for this study.

In the subgroup of nonimmunocompromised, 135 of total 178 survived, and 43 not survived. APACHE II score, SOFA score, proportion of Acute kidney injury, Hypertension, Procalcitonin, LDH, and whether performed mNGS (40.74\% vs $20.93 \%, P=0.018)$ showed significant difference. 
Table 8

Characteristics and baselines of patients who were survival or non-survival at day 28 in patients who were immunocompromised or not

\begin{tabular}{|c|c|c|c|c|c|c|}
\hline & \multicolumn{2}{|c|}{ Immunocompromised patient } & \multicolumn{4}{|c|}{ Nonimmunocompromised patient } \\
\hline & $\begin{array}{l}\text { Survival, (n } \\
=37)\end{array}$ & $\begin{array}{l}\text { Non- } \\
\text { survival, ( } \\
=13 \text { ) }\end{array}$ & $\begin{array}{l}p \\
\text { value }\end{array}$ & $\begin{array}{l}\text { Survival, (n } \\
=135)\end{array}$ & $\begin{array}{l}\text { Non- } \\
\text { survival, (n } \\
=43 \text { ) }\end{array}$ & $\begin{array}{l}p \\
\text { value }\end{array}$ \\
\hline Age(y) & $\begin{array}{l}55(39 \sim \\
62)\end{array}$ & $58(52 \sim 69)$ & 0.130 & $\begin{array}{l}66(53 \sim \\
72)\end{array}$ & $\begin{array}{l}62(48.5 \sim \\
74)\end{array}$ & 0.896 \\
\hline \multicolumn{7}{|l|}{ Sex } \\
\hline Female, $n(\%)$ & $13(35.1 \%)$ & $3(23.1 \%)$ & 0.423 & $41(30.4 \%)$ & $8(18.6 \%)$ & 0.133 \\
\hline Male, n(\%) & $24(64.9 \%)$ & $10(76.9 \%)$ & & $94(69.6 \%)$ & $35(81.4 \%)$ & \\
\hline APACHE II score & $\begin{array}{l}21(18 \sim \\
27)\end{array}$ & $\begin{array}{l}25.5(20.8 \sim ~ \\
27.8)\end{array}$ & 0.389 & $\begin{array}{l}18(14 \sim \\
23)\end{array}$ & $\begin{array}{l}24.5(19 \sim \\
38.5)\end{array}$ & $\begin{array}{l}\mathrm{P}< \\
0.001 *\end{array}$ \\
\hline SOFA score & $10(7 \sim 12)$ & $\begin{array}{l}12.5(7.25 \sim \\
15.3)\end{array}$ & 0.305 & $8(4 \sim 11)$ & $12(8 \sim 16)$ & $\begin{array}{l}P< \\
0.001 *\end{array}$ \\
\hline \multicolumn{7}{|l|}{$\begin{array}{l}\text { Major diagnosis of ICU } \\
\text { admission }\end{array}$} \\
\hline Severe pneumonia, $\mathrm{n}(\%)$ & $31(83.8 \%)$ & $13(100 \%)$ & 0.122 & $118(87.4 \%)$ & $36(83.7 \%)$ & 0.538 \\
\hline ARDS, n(\%) & $23(62.2 \%)$ & $10(76.9 \%)$ & 0.334 & $58(43.0 \%)$ & $16(37.2 \%)$ & 0.505 \\
\hline Septic shock, n(\%) & $5(13.5 \%)$ & $1(7.7 \%)$ & 0.578 & $21(15.6 \%)$ & $9(20.9 \%)$ & 0.412 \\
\hline Acute kidney injury, n(\%) & $1(2.7 \%)$ & $1(7.7 \%)$ & 0.430 & $15(11.1 \%)$ & $10(23.3 \%)$ & $0.046 *$ \\
\hline \multicolumn{7}{|l|}{ Comorbidities } \\
\hline Hypertension, n(\%) & $6(16.2 \%)$ & $1(7.7 \%)$ & 0.446 & $51(37.8 \%)$ & $9(20.9 \%)$ & $0.042^{*}$ \\
\hline Diabetes, n(\%) & $4(10.8 \%)$ & $2(15.4 \%)$ & 0.662 & $14(10.4 \%)$ & $4(9.3 \%)$ & 0.840 \\
\hline $\begin{array}{l}\text { Coronary artery disease, } \\
\mathrm{n}(\%)\end{array}$ & $2(5.4 \%)$ & $0(0 \%)$ & 0.392 & $17(12.6 \%)$ & $9(20.9 \%)$ & 0.178 \\
\hline Rheumatic disease, n(\%) & $10(27.0 \%)$ & $3(23.1 \%)$ & 0.780 & $8(5.9 \%)$ & $3(7.0 \%)$ & 0.803 \\
\hline $\begin{array}{l}\text { Chronic obstructive } \\
\text { pulmonary disease, n(\%) }\end{array}$ & $4(10.8 \%)$ & $1(7.7 \%)$ & 0.747 & $31(23.0 \%)$ & $8(18.6 \%)$ & 0.547 \\
\hline $\begin{array}{l}\text { Chronic kidney disease, } \\
\mathrm{n}(\%)\end{array}$ & $5(13.5 \%)$ & $2(15.4 \%)$ & 0.867 & $3(2.2 \%)$ & $3(7.0 \%)$ & 0.132 \\
\hline Chronic liver disease, $\mathrm{n}(\%)$ & $0(0 \%)$ & $0(0 \%)$ & - & $4(3.0 \%)$ & $3(7.0 \%)$ & 0.238 \\
\hline $\begin{array}{l}\text { Previous history of } \\
\text { malignancy, } n(\%)\end{array}$ & $9(24.3 \%)$ & $3(23.1 \%)$ & 0.928 & $31(23.0 \%)$ & $6(14.0 \%)$ & 0.205 \\
\hline
\end{tabular}




\begin{tabular}{|c|c|c|c|c|c|c|}
\hline \multirow[b]{2}{*}{ White Blood Cell $\left(\times 10^{9} / \mathrm{L}\right)$} & \multicolumn{3}{|c|}{ Immunocompromised patient } & \multicolumn{3}{|c|}{ Nonimmunocompromised patient } \\
\hline & $\begin{array}{l}7.2(0.1 \sim ~ \\
13.5)\end{array}$ & $\begin{array}{l}11.5(3.3 \sim \\
12.6)\end{array}$ & 1 & $\begin{array}{l}11.8(0.4 \sim \\
15.2)\end{array}$ & $\begin{array}{l}10.4(5.8 \sim \\
14.8)\end{array}$ & 0.356 \\
\hline Lymphocyte(× 109/L) & $\begin{array}{l}0.3(0.1 \sim \\
0.5)\end{array}$ & $\begin{array}{l}0.2(0.1 \sim \\
0.3)\end{array}$ & 0.460 & $\begin{array}{l}0.6(0.35 \sim ~ \\
1)\end{array}$ & $\begin{array}{l}0.6(0.3 \sim \\
1.2)\end{array}$ & 0.732 \\
\hline \multicolumn{7}{|l|}{ C-reactive protein $(\mathrm{g} / \mathrm{L})$} \\
\hline Procalcitonin(ng/mL) & $\begin{array}{l}0.8(0.3 \sim \\
4.5)\end{array}$ & $\begin{array}{l}2.9(0.4 \sim ~ \\
10.1)\end{array}$ & 0.381 & $\begin{array}{l}1.0(0.3 \sim \\
3.2)\end{array}$ & $\begin{array}{l}4.4(0.6 \sim \\
8.8)\end{array}$ & $0.005^{*}$ \\
\hline $\mathrm{LDH}(\mathrm{U} / \mathrm{L})$ & $\begin{array}{l}449(305.7 \\
\sim 613.9)\end{array}$ & $\begin{array}{l}541(404 \sim \\
679)\end{array}$ & 0.172 & $\begin{array}{l}308(240 \sim \\
453.1)\end{array}$ & $\begin{array}{l}495(289.2 \\
\sim 681)\end{array}$ & $0.002 *$ \\
\hline Perform mNGS, n(\%) & $8(21.6 \%)$ & $2(15.4 \%)$ & 0.629 & $55(40.7 \%)$ & $9(20.9 \%)$ & $0.018 *$ \\
\hline
\end{tabular}

\section{Note}

${ }^{*} \mathrm{P}<0.05$ was considered statistically significant.

Same as the analysis method of Table 5 , we select the indices present $\mathrm{P}<0.1$ for logistic regression. The analysis results show that the conclusion is almost consistent. Without performing mNGS, higher APACHA II score are high risk factors for the nonimmunocompromised subgroup, with the $\operatorname{Pr}(>|z|) 0.026$, and 0.005 .

Table 9

Logistic regression of indices $\mathrm{P}<0.1$ in Table 8

\begin{tabular}{|lllll|}
\hline & Estimate & Std. Error & z value & $\operatorname{Pr}(>|z|)$ \\
\hline APACHE II score & $9.2 \mathrm{E}-02$ & $3.2 \mathrm{E}-02$ & 2.84 & $0.005^{*}$ \\
\hline SOFA score & $2.7 \mathrm{E}-02$ & $6.0 \mathrm{E}-02$ & 0.45 & 0.656 \\
\hline Acute kidney injury & $6.6 \mathrm{E}-02$ & $6.1 \mathrm{E}-01$ & 0.11 & 0.912 \\
\hline Hypertension & $-9.9 \mathrm{E}-01$ & $5.3 \mathrm{E}-01$ & -1.88 & 0.060 \\
\hline Procalcitonin & $6.6 \mathrm{E}-03$ & $6.7 \mathrm{E}-03$ & 0.99 & 0.322 \\
\hline LDH & $7.0 \mathrm{E}-05$ & $8.0 \mathrm{E}-05$ & 0.88 & 0.380 \\
\hline Perform mNGS & $-1.1 \mathrm{E}+00$ & $5.1 \mathrm{E}-01$ & -2.23 & $\mathbf{0 . 0 2 6 *}$ \\
\hline
\end{tabular}

\section{Note}

${ }^{*} \mathrm{P}<0.05$ was considered statistically significant.

\section{Concordance Between mNGS and Culture}

The main sample types collected for pathogen identification are blood, BALF, sputum, only one sample was collected for testing in 63 cases, and the rest collected at least 2 samples, this produced a total of 157 samples, all samples except blood received both mNGS and culture. Sample of BALF (80), sputum (31), cerebrospinal 
fluid (1); and pleural effusion (4) were used for analysis. The pathogen distribution of mNGS result from 157 samples displayed in Fig. 2. The top 5 common bacteria were Acinetobacter baumannii (38/157, 24.2\%), Klebsiella pneumonia (24/157, 15.3\%), Stenotrophomonas maltophilia (23/157, 14.6\%), Enterococcus faecium (19/157,12.1\%), Pseudomonas aeruginosa (17/157, 10.8\%). Mycobacterium tuberculosis was detected in a case with BALF, compared with acid-fast bacterial stain and TB-DNA PCR nucleic amplification, both received positive result, which implied the potential of $\mathrm{mNGS}$ for Mycobacterium tuberculosis diaonosis. Fungi with high probability detected were Pneumocystis jirovecii $(17 / 157,10.8 \%)$ and three other species of Candida (30/157, $19.1 \%$ ). Pathogen distribution of patients received both mNGS and culture, which categorized as bacterial and fungal, listed in Table 10.

According to our statistics, mNGS and culture were both positive in 81 of total 116 samples (69.8\%). 4 were both negative (3.45\%), 29 samples were only positive in mNGS (25.0\%), and only 2 sample were positive by culture (1.72\%). For the double positive samples (81), the concordance between mNGS and culture were counted by match $(59,72.8 \%)$, partly match $(5,6.17 \%)$, mismatch $(17,21.0 \%)$, this illustrated in Fig. 2 . 
Table 10

Pathogen distribution in patients who received both mNGS and culture

\begin{tabular}{|c|c|c|c|c|}
\hline Microbial species & $\begin{array}{l}\text { Only positive in } \\
\text { mNGS, } n\end{array}$ & $\begin{array}{l}\text { Only positive in } \\
\text { culture, } n\end{array}$ & $\begin{array}{l}\text { Double } \\
\text { positive, } n\end{array}$ & $\begin{array}{l}\text { mNGS result } \\
\text { distribution }\end{array}$ \\
\hline \multicolumn{5}{|l|}{ Bacterial } \\
\hline $\begin{array}{l}\text { Acinetobacter } \\
\text { baumannii }\end{array}$ & 14 & 7 & 24 & $38(24.2 \%)$ \\
\hline Klebsiella pneumonia & 14 & 1 & 10 & $24(15.3 \%)$ \\
\hline $\begin{array}{l}\text { Stenotrophomonas } \\
\text { maltophilia }\end{array}$ & 11 & 5 & 12 & $23(14.6 \%)$ \\
\hline Enterococcus faecium & 18 & 0 & 1 & $19(12.1 \%)$ \\
\hline $\begin{array}{l}\text { Pseudomonas } \\
\text { aeruginosa }\end{array}$ & 12 & 5 & 5 & 17(10.8\%) \\
\hline $\begin{array}{l}\text { Staphylococcus } \\
\text { epidermidis }\end{array}$ & 13 & 1 & 1 & $14(8.9 \%)$ \\
\hline $\begin{array}{l}\text { Corynebacterium } \\
\text { striatum }\end{array}$ & 12 & 0 & 1 & $13(8.3 \%)$ \\
\hline Enterococcus faecalis & 12 & 1 & 0 & $12(7.6 \%)$ \\
\hline Staphylococcus aureus & 5 & 0 & 0 & $5(3.2 \%)$ \\
\hline $\begin{array}{l}\text { Burkholderia } \\
\text { multivorans }\end{array}$ & 3 & 1 & 1 & $4(2.5 \%)$ \\
\hline Nocardia farcinica & 0 & 0 & 1 & $1(0.6 \%)$ \\
\hline $\begin{array}{l}\text { Ralstonia } \\
\text { mannitolilytica }\end{array}$ & 0 & 0 & 1 & $1(0.6 \%)$ \\
\hline $\begin{array}{l}\text { Nontuberculosis } \\
\text { Mycobacteria }\end{array}$ & 4 & 0 & 0 & $5(3.2 \%)$ \\
\hline $\begin{array}{l}\text { Mycobacterium } \\
\text { tuberculosis }\end{array}$ & 1 & 0 & 0 & $1(0.6 \%)$ \\
\hline \multicolumn{5}{|l|}{ Fungal } \\
\hline Pneumocystis jirovecii & 17 & 0 & 0 & $17(10.8 \%)$ \\
\hline Candida albicans & 5 & 2 & 10 & $15(9.6 \%)$ \\
\hline Candida parapsilosis & 5 & 1 & 3 & $9(5.7 \%)$ \\
\hline Candida glabrata & 4 & 0 & 2 & $6(3.8 \%)$ \\
\hline Aspergillus sp. & 4 & 0 & 2 & $6(3.8 \%)$ \\
\hline
\end{tabular}

Clinical common herpes virus detected in this study were Cytomegalovirus (CMV, 17/157, 10.8\%), Herpes simplex virus (HSV,15/157, 9.6\%), and Epstein-Barr virus (EBV, 14/157, 8.9\%). For 21 cases, we conducted PCR test to confirm with mNGS (Table 11) [14-18], and the result revealed 100\% positive. 
Table 11

PCR test for virus detected by mNGS

\begin{tabular}{|c|c|c|c|}
\hline Virus & $\begin{array}{l}\text { Virus detected by } \\
\text { mNGS }\end{array}$ & PCR test result & Primers \\
\hline \multirow[t]{2}{*}{ CMV } & \multirow[t]{2}{*}{$17(10.8 \%)$} & \multirow[t]{2}{*}{$\mathrm{n}=13$, positive } & Forward: CTGTCGGTGATGGTCTCTTC \\
\hline & & & Reverse: CCCGACACGCGGAAAAGAAA \\
\hline \multirow[t]{2}{*}{ HSV } & \multirow[t]{2}{*}{$15(9.6 \%)$} & \multirow[t]{2}{*}{$\mathrm{n}=2$, positive } & Forward: CATCACCGACCCGGAGAGGGAC \\
\hline & & & Reverse: GGGCCAGGCGCTTGTTGGTGTA \\
\hline \multirow[t]{2}{*}{ EBV } & \multirow[t]{2}{*}{$14(8.9 \%)$} & \multirow[t]{2}{*}{$\mathrm{n}=2$, positive } & Forward: TGTGAACCTTATGGAGATGTGC \\
\hline & & & Reverse: CACTGGAAATGCTTGGTCAATA \\
\hline \multirow[t]{2}{*}{ Hepatitis $B$ virus } & \multirow[t]{2}{*}{$6(3.8 \%)$} & \multirow[t]{2}{*}{$\mathrm{n}=2$, positive } & Forward: GTGTCTGCGGCGTTTTATCA \\
\hline & & & Reverse: GACAAACGGGCAACATACCTT \\
\hline \multirow{2}{*}{$\begin{array}{l}\text { Influenza A } \\
\text { Virus }\end{array}$} & \multirow[t]{2}{*}{$2(1.3 \%)$} & \multirow[t]{2}{*}{$\mathrm{n}=2$, positive } & Forward: CACCCCAGAAATAGCCAAAA \\
\hline & & & Reverse: TCCTGATCCAAAGCCTCTAC \\
\hline
\end{tabular}

\section{Note}

n, number of cases conducted PCR test with virus positive result in mNGS; CMV, Cytomegalovirus; HSV, Herpes simplex virus; EBV, Epstein-Barr virus.

Compared with culture, the rapid feedback of mNGS and high positive rate hasten clinical decision making and guide clinical laboratories to improve culture conditions for fastidious organisms. Moreover, for coinfections, unculturable microorganism infections, and new pathogenic infections, mNGS shows great proven advantages $[19,20]$.

\section{Discussion}

In our single center retrospective study, patients in mNGS group had a higher proportion of ARDS, lower absolute number of peripheral blood lymphoid bacteria, higher LDH level, and higher APACHE II score (although there was no statistical difference between the two groups). This indicating that in clinical work, we prefer to mNGS detection for patients with more severe disease severity and lower immune level [21]. In contrast, the 28day mortality rate of the patients in the mNGS group was lower after the baseline difference correction, indicating that mNGS could improve the prognosis of patients in ICU $[22,23]$. Moreover, subgroup analysis showed that mNGS could improve the 28-day mortality of nonimmunosuppressive patients. While for immunosuppressive patients, the expected conclusion has not been reached. That's probably because the cases of nonimmunosuppressive patients are not enough in this retrospective study, and more cases of immunosuppressive patients are needed for further clarifying [24].

Logistic regression showed that, not performing mNGS, high APACHE II score and hypertension were independent risk factors for 28-day mortality, which strongly suggested that mNGS was one of the key factors affecting prognosis [23]. The improvement of prognosis may be related to the sensitivity of mNGS to pathogen 
detection. Despite prolonged hospital stay and increased costs, this is more likely to be associated with the severity of the illness.

mNGS can improve the sensitivity of pathogens detection, including bacteria, fungi, viruses and parasite are more sensitive than the conventional culture and PCR methods at the same time. Although it is still unable to distinguish whether the pathogens detected by mNGS are pathogenic microorganisms, combined with prognostic indicators, it can provide more enlightenment for clinical work [4-7]. Theoretically, it can detect all pathogens in a clinical sample unbiased at one time, and shows proven advantages for rare, novel, difficult cases of infectious diseases. However, there is still a lot of improvements for this technique, for example, to formulate negative and positive criteria, improve the turnaround time and optimize the method for drug resistance prediction for clinical.

For different sample type like blood, sputum, cerebrospinal fluid, tissue, swab and other miscellaneous specimen, how to establish a unified nucleic acid extraction standard makes the detection efficiency of pathogens maximization difficult. Host nucleic acid contamination is also a major factor that interferes with pathogen detection. In the process of nucleic acid extraction, how to remove host effectively, thereby enrich pathogen nucleic acid and improve the sensitivity of $\mathrm{mNGS}$, this still needs relevant research to be solved. In terms of bioinformatics, recent studies have evaluated the merits and demerits of different tools. The existing methods not only have different algorithms, but also use different databases, which leads to differences in pathogen identification ability and relative abundance calculation [25]. In the absence of a unified standard in the process of mNGS, the user's choice of the software based on personal experience, accessibility and convenience will affect the repeatability and reliability of the results, which will become an obstacle to the clinical standardization of mNGS.

This technique takes a long time comparatively, usually 24 to 48 hours of turnaround time at present. This needs to be further improved to meet the needs of ICU. In addition, the application of mNGS for patient in ICU is suitable for carrying out in the hospital, while expensive instruments and difficulty of operation present to be a problem.

Presently, mNGS is mainly based on short read length sequencing, for the characteristics of sequencing platforms (Illumina, Thermo Fisher, MGISEQ). In 2014, Hasman et al clarified that mNGS can be used for the detection of pathogens and drug-resistant genes in urine samples [26]. However, samples with low abundance of microbial sequences, it is difficult to determine the attribution of the drug-resistant genes accurately. Improvement of this technique is expected in the future so as to provide proven precise medical service for infectious pathogens identification for clinical practice.

\section{Conclusion}

We found that more pathogens could be detected using mNGS compared to traditional microbial detection methods. mNGS may improve the prognosis and reduce the 28-day mortality rate of patients with infectious diseases on mechanical ventilation in ICU. This technique has shown its advantages comparing with conventional methods, and will be wildly used as a promising technology for infectious disease. Additional studies are required to confirm the usefulness of this technology.

Page 21/28 


\section{Declarations}

\section{Ethical Approval and Consent to participate}

The study was approved by the Institutional Ethics Committee of the First Affiliated Hospital of Guangzhou Medical University(Number:2020K-42). Informed consent was waived by the ethics committee due to the retrospective nature of study design.

\section{Consent for publication}

All of the participating patients consented to the publication of their clinical data.

\section{Availability of supporting data}

The datasets analyzed during the current study are available from the corresponding author on reasonable request.

\section{Competing interests}

All authors declare that they have no competing interests.

\section{Funding}

The study was funded by the National Science and Technology Major Project (No.2017ZX10204401), National Natural Science Foundation of China (No.81970071) and Natural Science Foundation of Guangdong Province, China (2020A1515011459).

\section{Authors' contributions}

YX and LS contributed to the conception and design of the study, acquisition of data, analysis and interpretation of data, and drafted the manuscript. YDX and YML contributed to the analysis and interpretation of data and revision of the manuscript. All other authors participated in the analysis and interpretation of data and drafted the manuscript. All authors read and approved the final manuscript.

\section{Acknowledgements}

We thank all patients and clinicians from the First Affiliated Hospital of Guangzhou Medical University in Guangzhou, China, for their cooperation and participation in the study.

\section{References}

1. Lanks CW, Musani Al, Hsia DW, et al. Community-acquired Pneumonia and Hospital-acquired Pneumonia. Med Clin North Am. 2019;103(3):487-501.

2. Wan $\mathrm{K}$, Liang $\mathrm{H}, \mathrm{Yan} \mathrm{G}$, et al. A quality assessment of evidence-based guidelines for the prevention and management of ventilator-associated pneumonia: a systematic review. J Thorac Dis. 2019;11(7):2795807.

3. Restrepo MI, Mortensen EM, Rello J, et al. A. Late admission to the ICU in patients with communityacquired pneumonia is associated with higher mortality. Chest. 2010;137(3):552-7. 
4. Wilson MR, Naccache SN, Samayoa E, et al. Actionable diagnosis of neuroleptospirosis by next-generation sequencing. N Engl J Med. 2014;370(25):2408-17.

5. Chiu CY, Miller SA, et al. Clinical metagenomics. Nat Rev Genet. 2019;20(6):341-55.

6. Simner PJ, Miller S, Carroll KC, et al. Understanding the Promises and Hurdles of Metagenomic NextGeneration Sequencing as a Diagnostic Tool for Infectious Diseases. Clin Infect Dis. 2018;66(5):778-88.

7. Xie Y, Du J, Jin W, et al. Next generation sequencing for diagnosis of severe pneumonia: China, 20102018. J Infect. 2019;78(2):158-69.

8. Ai JW, Weng SS, Cheng Q, et al. Human Endophthalmitis Caused By Pseudorabies Virus Infection, China, 2017. Emerg Infect Dis. 2018;24(6):1087-90.

9. Fan S, Ren H, Wei Y, et al. Next-generation sequencing of the cerebrospinal fluid in the diagnosis of neurobrucellosis. Int J Infect Dis. 2018;67:20-4.

10. Li H, Gao H, Meng H, et al. Detection of Pulmonary Infectious Pathogens From Lung Biopsy Tissues by Metagenomic Next-Generation Sequencing. Front Cell Infect Microbiol. 2018;8:205.

11. Miao Q, Ma Y, Wang Q, et al. Microbiological Diagnostic Performance of Metagenomic Next-generation Sequencing When Applied to Clinical Practice. Clin Infect Dis. 2018;67(suppl_2):231-40.

12. Bittinger $\mathrm{K}$, Charlson ES, Loy $\mathrm{E}$, et al. Improved characterization of medically relevant fungi in the human respiratory tract using next-generation sequencing. Genome Biol. 2014;15(10):487.

13. Schlaberg R, Chiu CY, Miller S, et al Professional Practice Committee and Committee on Laboratory Practices of the American Society for Microbiology; Microbiology Resource Committee of the College of American Pathologists. Validation of metagenomic next-generation sequencing tests for universal pathogen detection. Arch Pathol Lab Med. 2017;141(6):776-86.

14. Boriskin YS, Rice PS, Stabler RA, et al. DNA microarrays for virus detection in cases of central nervous system infection. J Clin Microbiol. 2004;42(12):5811-8.

15. Coisel Y, Bousbia S, Forel JM, et al. Cytomegalovirus and herpes simplex virus effect on the prognosis of mechanically ventilated patients suspected to have ventilator-associated pneumonia. PLoS One. 2012;7(12):e51340.

16. Fernando S, Booth J, et al. Association of cytomegalovirus infection with post-transplantation cardiac rejection as studied using the polymerase chain reaction. J Med Virol. 1994;42(4):396-404.

17. Mendy ME, Kaye S, van der Sande M, et al. Application of real-time PCR to quantify hepatitis B virus DNA in chronic carriers in The Gambia. Virol J. 2006;3:23.

18. Panning M, Eickmann M, Landt O, et al. Detection of influenza $A(H 1 N 1)$ v virus by real-time RT-PCR. Euro Surveill. 2009;14(36):19329.

19. Rhodes J, Hyder JA, Peruski LF, et al. Antibiotic use in Thailand: quantifying impact on blood culture yield and estimates of pneumococcal bacteremia incidence. Am J Trop Med Hyg. 2010;83(2):301-6.

20. Gosiewski T, Ludwig-Galezowska AH, Huminska K, et al. Comprehensive detection and identification of bacterial DNA in the blood of patients with sepsis and healthy volunteers using next-generation sequencing method-the observation of DNAemia. Eur J Clin Microbiol Infect Dis. 2017;36(2):329-36.

21. Burillo A, Bouza $E$, et al. Use of rapid diagnostic techniques in ICU patients with infections. BMC Infect Dis. 2014;14:593. 
22. Liang $\mathrm{J}$, $\mathrm{Li} \mathrm{Z}$, Dong $\mathrm{H}$, et al. Prognostic factors associated with mortality in mechanically ventilated patients in the intensive care unit: A single-center, retrospective cohort study of 905 patients. Medicine. 2019;98(42):e17592.

23. Paiva JA, Pereira JM, Tabah A, et al. Characteristics and risk factors for 28-day mortality of hospital acquired fungemias in ICUs: data from the EUROBACT study. Crit Care. 2016;20:53.

24. Han D, Li Z, Li R, et al. mNGS in clinical microbiology laboratories: on the road to maturity. Crit Rev Microbiol. 2019;45(5-6):668-85.

25. Ye SH, Siddle KJ, Park DJ, et al. Benchmarking Metagenomics Tools for Taxonomic Classification Cell. 2019;178(4):779-94.

26. Hasman H, Saputra D, Sicheritz-Ponten T, et al. Rapid whole-genome sequencing for detection and characterization of microorganisms directly from clinical samples. J Clin Microbiol. 2014;52(1):139-46.

\section{Figures}

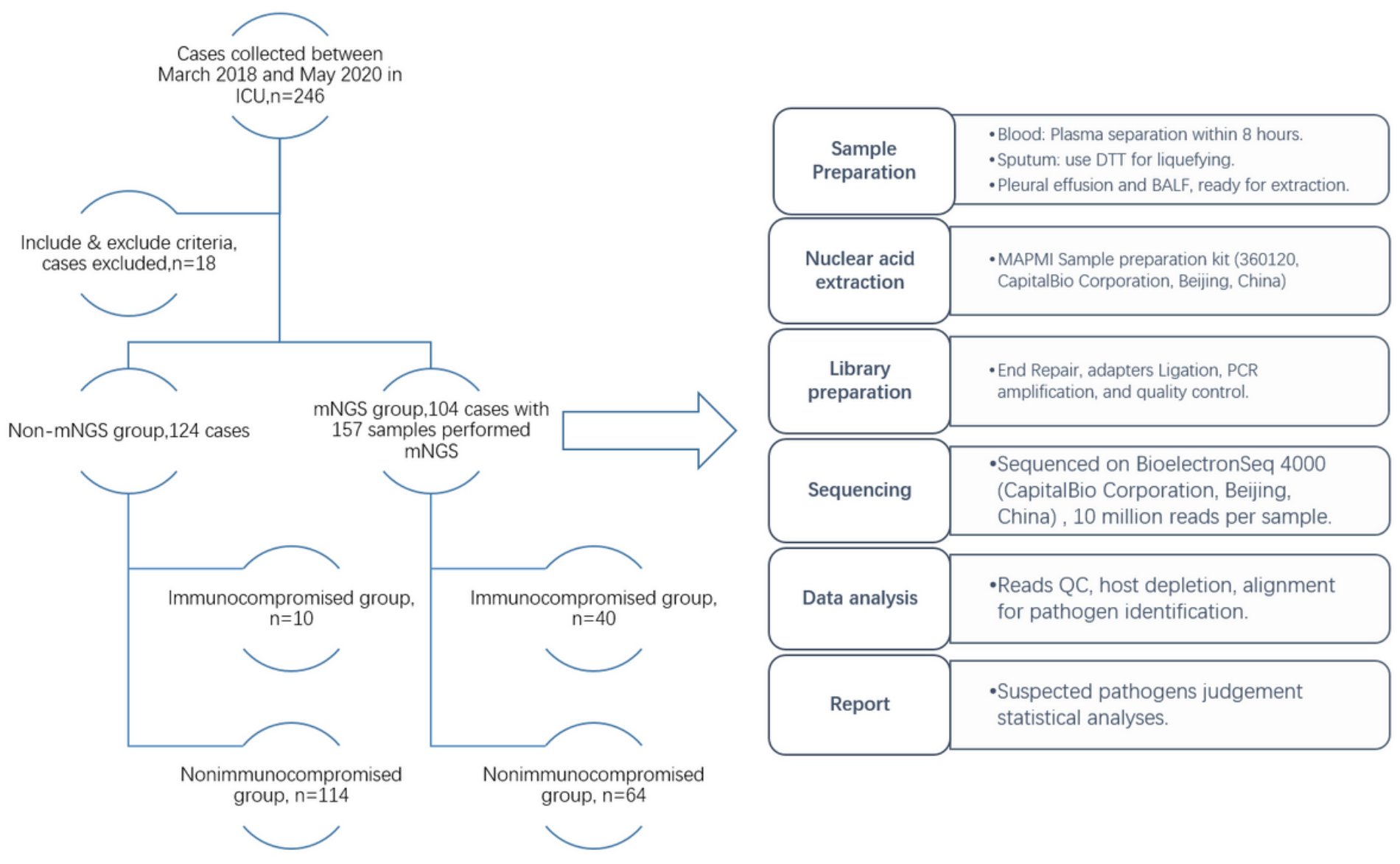

\section{Figure 1}

The flowchart of case inclusion and exclusion, with a total of 246 cases admitted to ICU, 224 cases were enrolled in this study. These cases divided into mNGS group, non-mNGS group, immunocompromised subgroup, and nonimmunocompromised subgroup. 157 samples used for further analysis while 116 samples both received mNGS and culture result. 


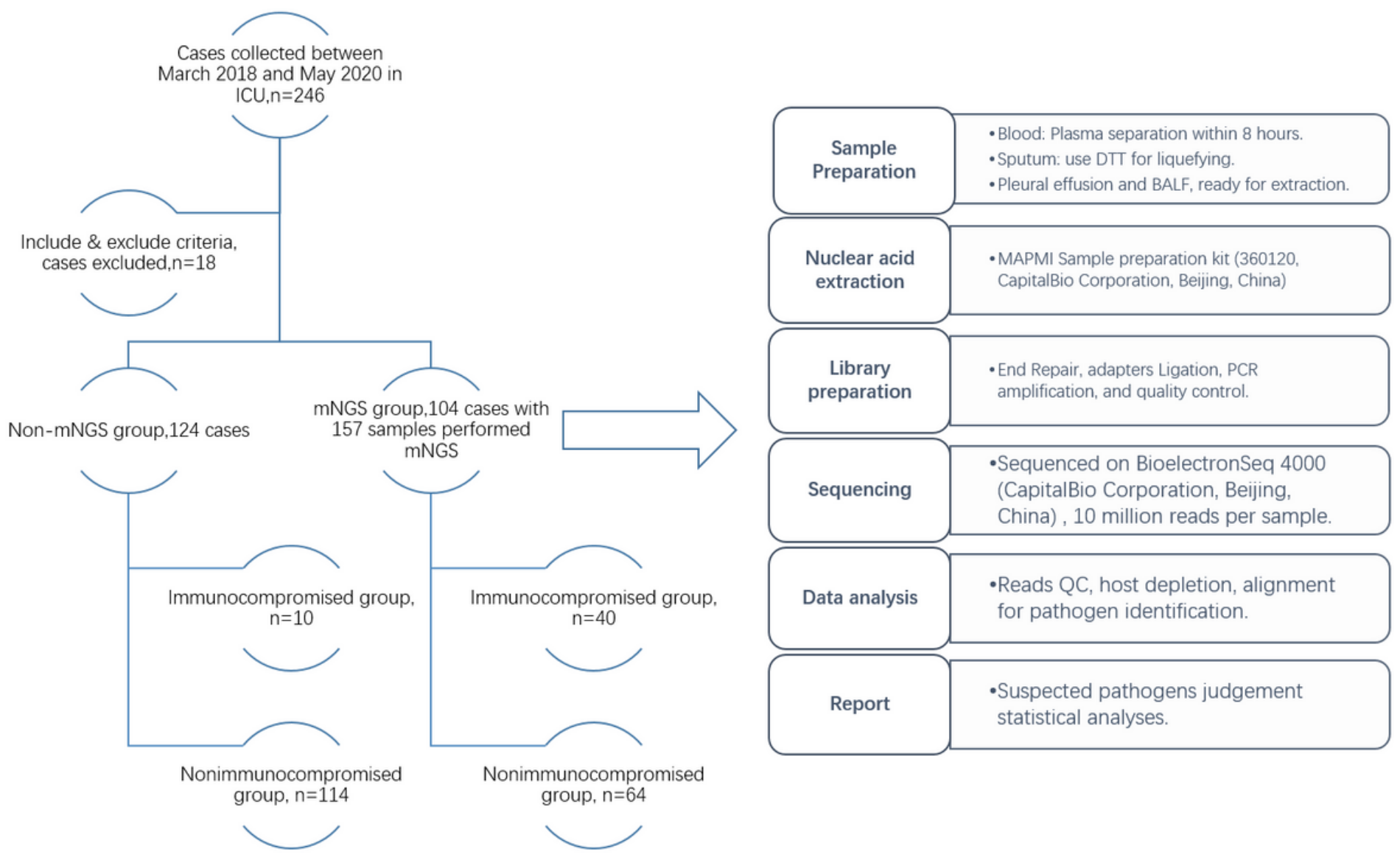

\section{Figure 1}

The flowchart of case inclusion and exclusion, with a total of 246 cases admitted to ICU, 224 cases were enrolled in this study. These cases divided into mNGS group, non-mNGS group, immunocompromised subgroup, and nonimmunocompromised subgroup. 157 samples used for further analysis while 116 samples both received mNGS and culture result. 


\section{Pathogen Distribution of mNGS}

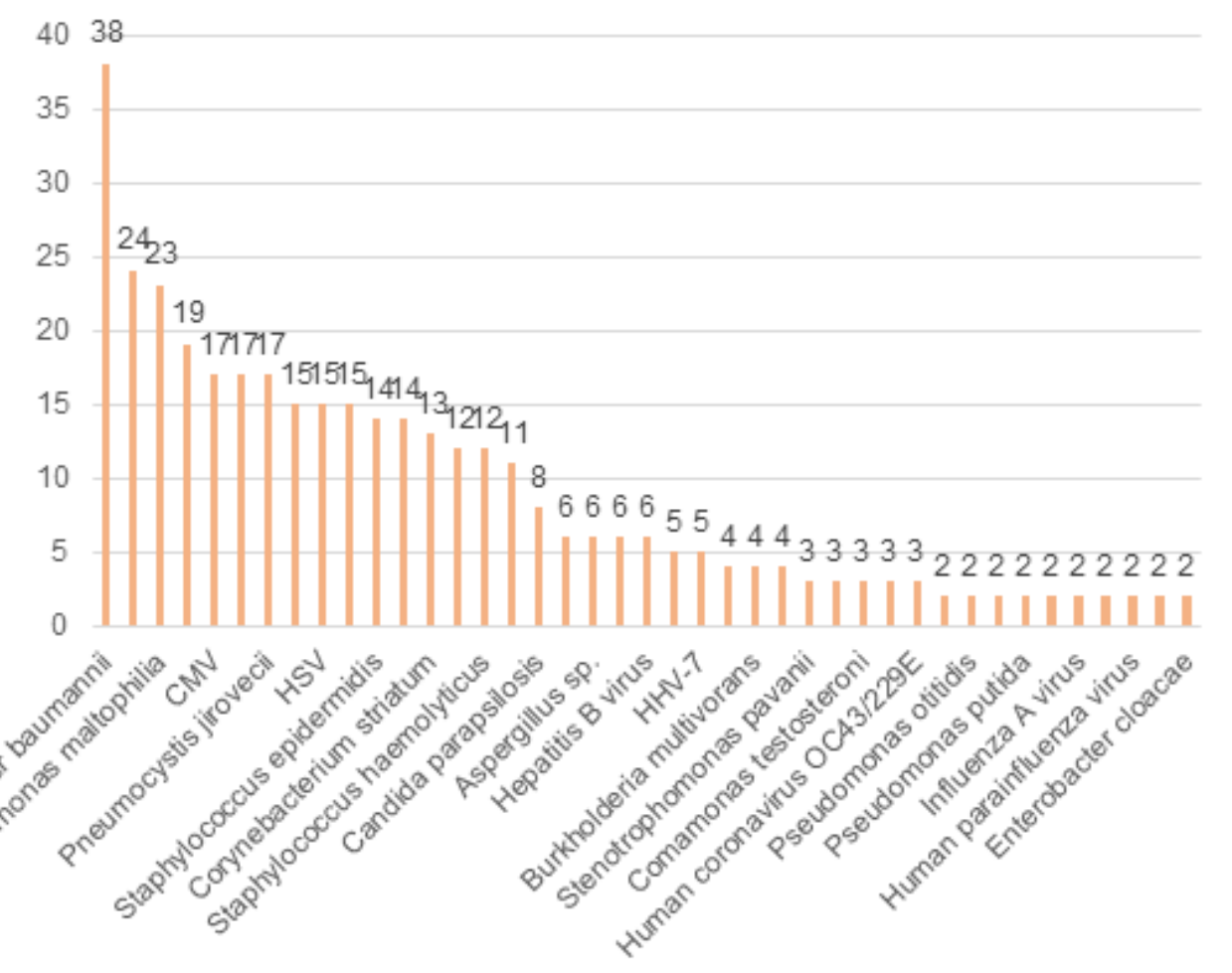

\section{Figure 2}

Pathogen distribution of mNGS result. With 38 of 157 samples found Acinetobacter baumannii by mNGS, and the results detected less than 2 were not displayed in illustration. 


\section{Pathogen Distribution of mNGS}

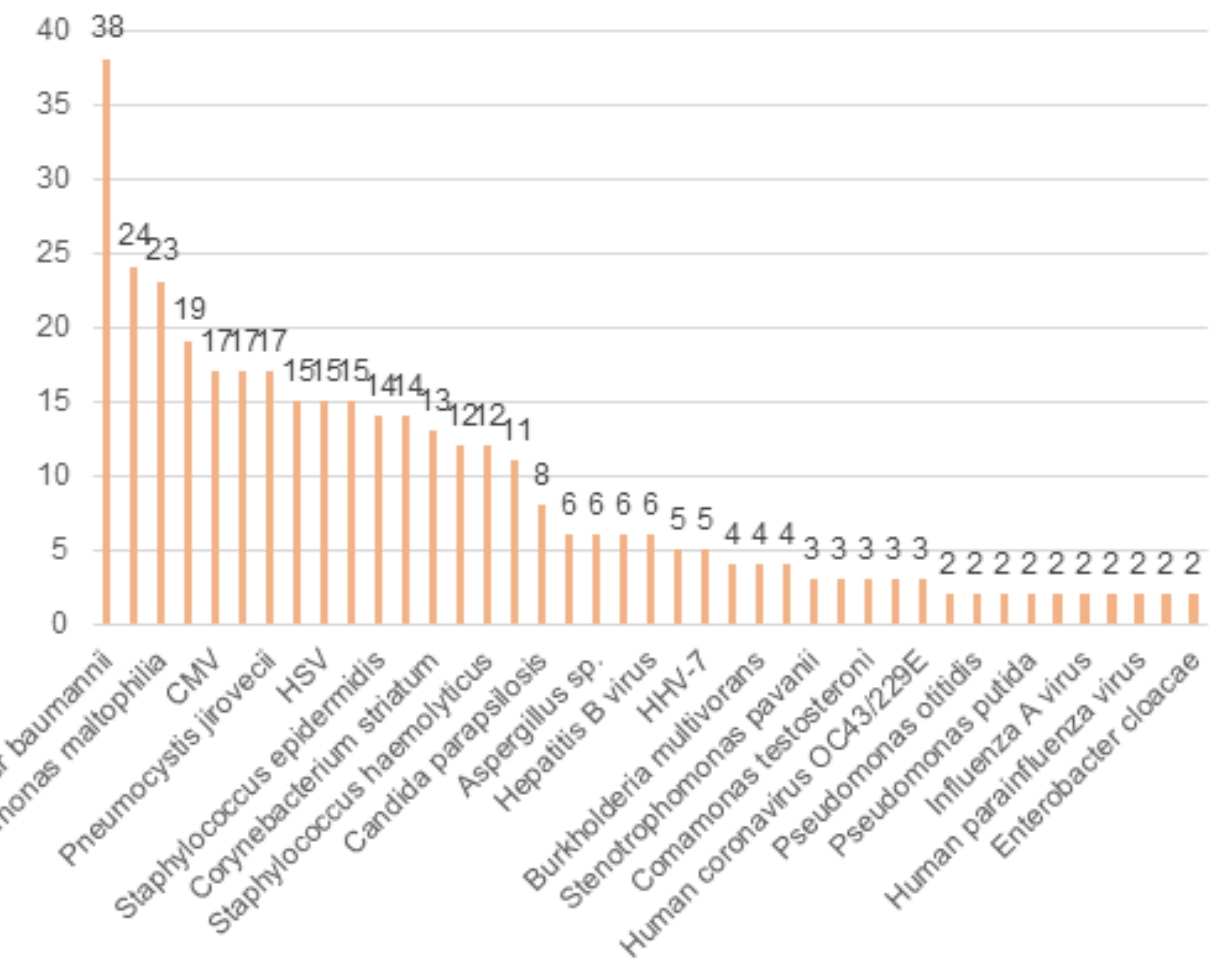

\section{Figure 2}

Pathogen distribution of mNGS result. With 38 of 157 samples found Acinetobacter baumannii by mNGS, and the results detected less than 2 were not displayed in illustration.

\section{Concordance Between mNGS and Culture}

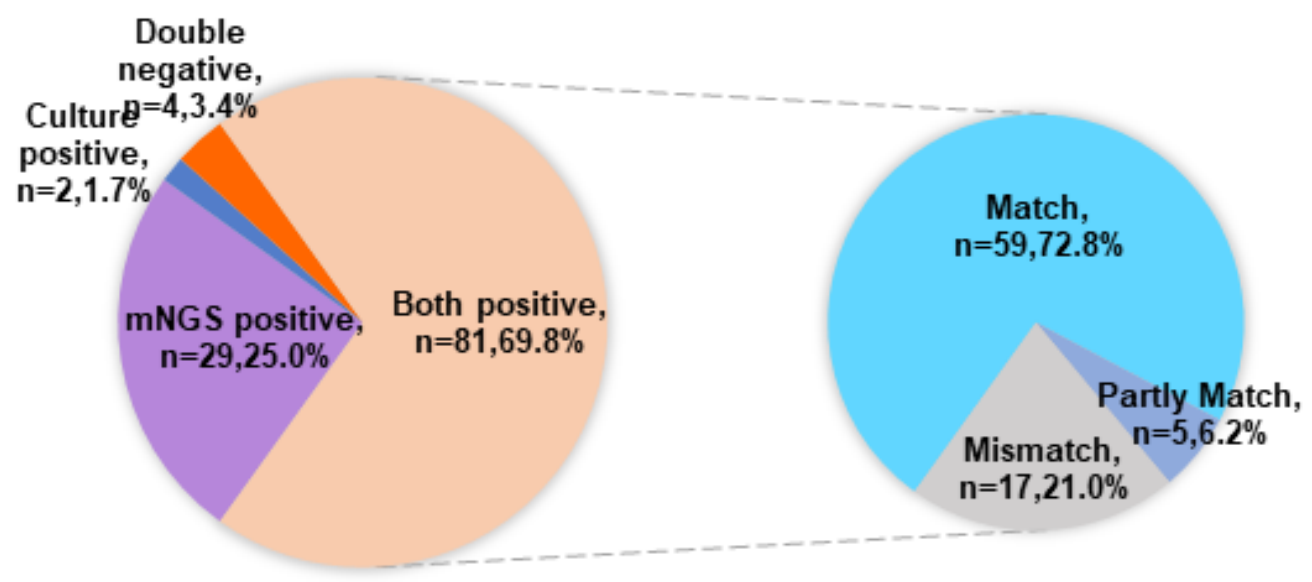

\section{Figure 3}

Pie chart illustrates the positivity distribution of mNGS and culture for 116 samples. 81 (69.8\%) samples were both positive by mNGS and culture, 59 (match, $72.8 \%$ ) of which revealed pretty concordance, 3 (partly match, 
6.2\%) samples got overlapping, while 19 (mismatch, 21.0\%)samples conflict between mNGS and culture.

\section{Concordance Between mNGS and Culture}

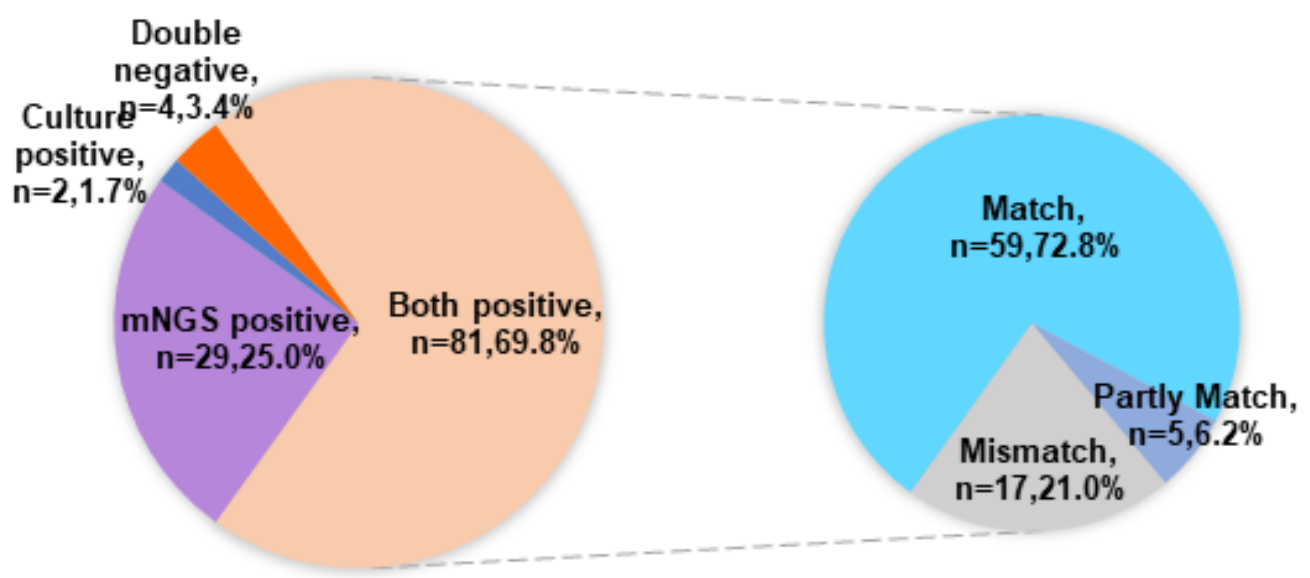

\section{Figure 3}

Pie chart illustrates the positivity distribution of mNGS and culture for 116 samples. 81 (69.8\%) samples were both positive by mNGS and culture, 59 (match, 72.8\%) of which revealed pretty concordance, 3 (partly match, $6.2 \%$ ) samples got overlapping, while 19 (mismatch, 21.0\%)samples conflict between mNGS and culture. 